\title{
The Role of Age on Multisensory Bodily Experience: An Experimental Study with a Virtual Reality Full-Body Illusion
}

\author{
Silvia Serino, $\mathrm{PhD}^{1,2}$ Federica Scarpina, $\mathrm{PhD}, 3$ Antonios Dakanalis, $\mathrm{PhD}^{4,5}$ Anouk Keizer, $\mathrm{PhD}$, \\ Elisa Pedroli, BA, ${ }^{2}$ Gianluca Castelnuovo, PhD, ${ }^{1,3}$ Alice Chirico, BA, Valentina Catallo, PhD, \\ Daniele di Lernia, BA, and Giuseppe Riva, $\mathrm{PhD}^{1,2}$
}

\begin{abstract}
A growing body of evidence demonstrated that it is feasible to induce ownership over an artificial body to alter bodily experience. However, several uncharted aspects about full-body illusion applications need to be tackled before a complete exploitation of these methods in clinical practice. This work is devoted to explore possible individual age-related differences in shaping changes in body representations induced with a full-body illusion. A total of 40 women were divided into two different age groups according to the median of the variable age. Participants estimated the width of three different body parts (i.e., shoulders, abdomen, and hips) before the entire illusion was induced (baseline), and after the synchronous and the asynchronous conditions. Results revealed that 26-to-55-year-old participants were more resistant to changes induced by the bodily illusion, whereas 19-to-25-year-old participants underestimated their bodies after both conditions. The findings were discussed in terms of the literature exploring age differences in responses to bodily illusion, which could suggest a Bayesian mechanism underlying these individual differences.
\end{abstract}

Keywords: bodily illusion, virtual reality, body image, eating disorders

\section{Introduction}

$\mathbf{I}$ N RECENT YEARS, A growing body of studies has highlighted the potentiality of the "bodily illusions" for altering body representations, namely participants feel significantly fatter or thinner than they really are..$^{1-3}$ A "bodily illusion" can be defined as an experimental setup able to manipulate the experience of being in a body through delivering a synchronized multisensory stimulation (for reviews, see Refs. ${ }^{4-7}$ ). One of the most prominent techniques allowing participants to feel that they are the owners of another (whole) body is the fullbody illusion. The illusory ownership over an artificial body (i.e., a mannequin or a virtual avatar) is achieved by observing from a first-person perspective how the artificial body is being stroked while a synchronous tactile input is perceived on the actual body. ${ }^{8-11}$
For example, when an illusionary embodiment over a virtual body with an enlarged abdomen was induced in young men, they congruently judged themselves to have larger abdomen size. ${ }^{2}$

Evidence deriving from the extant experimental studies for a (a) direct link between perceptual (in terms of a difficulty to provide an accurate estimation of own body size) and affective (in terms of body dissatisfaction) components of body representation disturbances, ${ }^{3}$ and (b) a positive affective response with the full-body illusion modulated by eating disorder psychopathology, ${ }^{3}$ may suggest clinical applications for this method. ${ }^{12,13}$ Indeed, the potentiality of the full-body illusion in decreasing the overestimation of body size in patients with anorexia nervosa ${ }^{14}$ and in modulating body representation disturbances in severe and nonoperable obesity patients with regular binge-eating behaviors ${ }^{15}$ was recently reported in the literature.

\footnotetext{
${ }^{1}$ Department of Psychology, Università Cattolica del Sacro Cuore, Milan, Italy.

${ }^{2}$ Applied Technology for Neuro-Psychology Lab, IRCCS Istituto Auxologico Italiano, Milan, Italy.

${ }^{3}$ Psychology Research Laboratory, IRCCS Istituto Auxologico Italiano, Ospedale San Giuseppe, Piancavallo, Italy.

${ }_{5}^{4}$ Department of Brain and Behavioral Sciences, University of Pavia, Pavia, Italy.

${ }^{5}$ Department of Medicine and Surgery, University of Milano-Bicocca, Monza, Italy.

${ }^{6}$ Department of Experimental Psychology, Faculty of Social and Behavioural Sciences, Utrecht University, Utrecht, Netherlands.
}

(C) Silvia Serino et al. 2018; Published by Mary Ann Liebert, Inc. This Open Access article is distributed under the terms of the Creative Commons License (http://creativecommons.org/licenses/by/4.0), which permits unrestricted use, distribution, and reproduction in any medium, provided the original work is properly cited. 
A recent work analyzing available systematic reviews and meta-analyses about virtual reality (VR) in healthcare clearly indicated the potentiality of this technology not only in eating and weight disorders but also in other clinical conditions, such as anxiety disorders and chronic pain. ${ }^{16}$ As regards eating and weight disorders, VR-based protocols for cue exposure to food stimuli ${ }^{17,18}$ and body image concerns ${ }^{19}$ seem to be effective in reducing eating disorder symptoms. Specifically, it is suggested the exploitation of full-body illusions to further enhance the efficacy of current treatments of body representaton disturbances. ${ }^{16,20}$

However, several uncharted aspects about full-body illusion applications need to be tackled before a complete exploitation of this method in clinical practice. Thus, this work explores possible individual age-related differences in modulating changes of body representation induced with a VR full-body illusion. Indeed, the majority of the aforementioned studies ${ }^{1,2}$ describing changes in body perception (induced by the fullbody illusion) were carried out on healthy samples of young women (i.e., mean age of 25 years).

This exploratory study uses a VR full-body illusion ${ }^{1,14}$ to induce illusory ownership over a virtual body with a skinny abdomen in healthy women of different ages to investigate the relationship between age and the magnitude and direction of the changes in body representation.

\section{Materials and Methods}

\section{Participants}

Forty women took part in this study. Participants were recruited through convenience and snowball sampling-in particular, students from the Catholic University of Milan were invited during lessons and asked to refer friends. No economical compensation was given. Inclusion criteria were being female, having between 18 and 55 years of age, having no current or history of neurological illness, and no current physical conditions known to influence body weight/size (for example, pregnancy). In addition, participants were required to have a body mass index (BMI) between 18.5 and $25 \mathrm{~kg} / \mathrm{m}^{2}$ and not to have a current or history of psychiatric illness (as defined in the DSM-IV-TR, Axis I). ${ }^{21}$

Participants were divided into two different age groups according to the median of the variable "age": age group 1 (range: 19-25 years old, mean age of 22.55 years [standard deviation, $S D=1.82]$, mean BMI of $\left.21.44 \mathrm{~kg} / \mathrm{m}^{2}[S D=2.06]\right)$ and age group 2 (range: $26-55$ years old, mean age of 40.20 years $[S D=10.64]$, mean BMI of $\left.21.35 \mathrm{~kg} / \mathrm{m}^{2}[S D=2.16]\right)$. The two age groups did not differ in terms of BMI $[t(38)=0.121 ; p=0.904]$.

\section{Procedure}

All participants provided written informed consent for participating in the study. This study was approved by the Ethics Review Board of the Catholic University of the Sacred Heart (Milan, Italy). At the start of the experiment, participants completed a body size estimation task ${ }^{14}$ by estimating the width of their shoulders, abdomen, and hips ("preillusion estimation"). Subsequently, they were required to wear the HMD-Oculus Rift DK2 to experience the full-body illusion. The virtual body of a woman ( $\sim 25$ years old, which corresponds to the median age of our sample) with a skinny abdomen (i.e., with a different shape/size in comparison with the actual body of participants) standing upright in a stimulus-free room was used to induce the fullbody illusion. 14 The virtual room was developed with the software Unity $3 \mathrm{D},{ }^{\mathrm{a}}$ whereas the avatar was modeled using the software MakeHuman. ${ }^{\text {b }}$

The waist circumference of the avatar was $73.95 \mathrm{~cm}$, contrasting the mean waist circumference of both the age group $1[t(19)=-7.078 ; p<0.001 ;$ mean $=84.11 ; S D=8.34)]$ and age group $2[t(19)=-7.749 ; p<0.001 ;$ mean $=79.23$; $S D=10.45)$, whereas there was no significant difference in waist circumference between the two groups $[t(38)=1.633$; $p=0.111)]$. The full-body illusion (for more details, see Fig. 1) was induced twice after the procedure of previous studies ${ }^{1,14}$ : (a) a synchronous visuotactile stimulation (i.e., the experimental condition) and (b) an asynchronous visuotactile stimulation (i.e., the control condition). Both conditions were delivered in a counterbalanced order, following a within-subject design. Both stimulations lasted 90 seconds, a time interval sufficient to induce the illusion as previously reported. ${ }^{1,14}$

In the synchronous visuotactile stimulation, the experimenter provided a tactile stimulation on the participants' abdomen for 90 seconds with the brush attached to the motion-tracking device (Razer Hydra). Specifically, there was a synchronous stimulation between the visual input (i.e., a virtual hand holding a brush stroking the abdomen of the virtual body) and what they perceived on their own body. Instead, in the asynchronous visuotactile stimulation, there was a delay between the tactile stimulation on the participant's abdomen and the visual input. Indeed, the touch on participants' abdomen was actually recorded by pressing a button on the Razer Hydra at the beginning of the movement. This procedure stopped the image seen by the participants as soon as the touch ended, and then it was replayed in VR when the experimenter finished each touch. After these virtual experiences, participants performed again the body size estimation task ("postillusion estimation"). They also completed the Embodiment Questionnaire, after each illusionary (synchronous/asynchronous) condition.

\section{Body size estimation task}

To investigate whether illusionary ownership over a virtual body with a skinny abdomen would result in changes in body representations between the two groups, participants were required to provide an estimation of the width of three different body parts, namely shoulders, abdomen, and hips. Participants stood in front of a wall and they were asked to estimate the horizontal distance between the left and right side of each body part placing adhesive stickers on the wall. They were explicitly asked to not look at their own body during the task. The body size estimation task was performed three times (before the entire full-body illusion, after the synchronous visuotactile stimulation, after the asynchronous visuotactile stimulation). The actual width of the body parts was measured at the end of the experiment.

\section{Embodiment Questionnaire}

A short (15-item) Embodiment Questionnaire, ${ }^{1,22}$ rated on a 7 point Likert scale (ranging from 1, fully disagree to 7, fully agree), was administered after completing each 


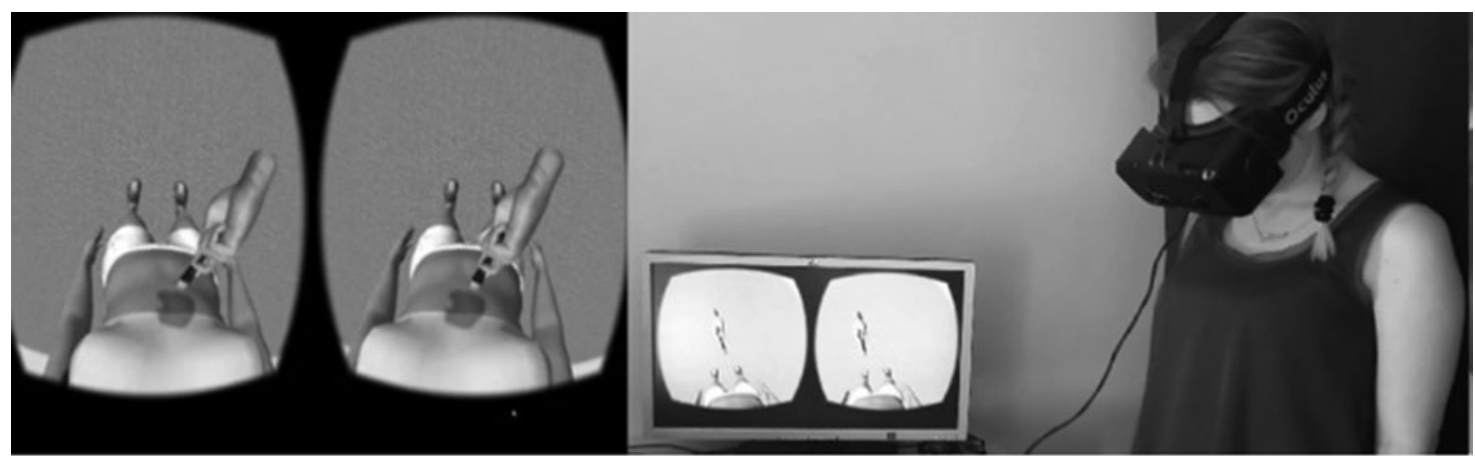

- During the illusion, participants were asked to wear a head mounted display (HMD, Oculus Rift DK2) connected to a portable computer (HP TRUE VISION with CPU Intel $\$$ Core ${ }^{\mathrm{TM}} \mathrm{i} 7$ ), allowing visualization of the virtual body (1080p resolution). A motiontracking device (Razer Hydra), connected to the portable computer, was used to visually mimic the stroking movements on the abdomen of the body.

- During both conditions (synchronous visuo-tactile stimulation vs. asynchronous visuo-tactile stimulation), all participants were required to bare their abdomen and to stand upright in the same position as the virtual body (i.e., first-person perspective) and to stand still and look down with the head mounted display, as they had to look at their actual abdomen in order to see the abdomen of the virtual body and experiment the illusion.

FIG. 1. The VR body swap illusion. The illusory ownership over the virtual body ( $\sim 25$ years old) is achieved by observing from a first-person perspective how the virtual body is being touched on the abdomen while a synchronous input is perceived on the actual body. VR, virtual reality.

condition (i.e., synchronous vs. asynchronous visuotactile stimulation) to assess the extent to which participants experienced the full-body illusion on three different components. The first one measured the body ownership (e.g., "I felt as the virtual body was my body"); the second, the selflocation (e.g., "I felt as I was inside the virtual body"); and the third, the sense of agency (e.g., "I had the feeling that I had the control over the virtual body"). The three components were obtained by calculating the mean scores from the items.

\section{Results}

\section{Embodiment Questionnaire}

A mixed analysis of variance (ANOVA) with group and condition was carried out for each of the three subscales of the Embodiment Questionnaire (i.e., ownership, self-location, and sense of agency). Concerning ownership, a main effect of condition emerged (Table 1), with significantly higher scores in the synchronous condition than in the asynchronous condition ( $p=0.043$ ). A same pattern of results emerged for self-location, with higher scores in the synchronous condition than in the asynchronous condition $(p=0.002)$. Concerning sense of agency, no significant differences were found between the two conditions. Neither a main effect of group nor interactions between group and condition were found (see Table 1 for full statistics) for the three measures (i.e., ownership, self-location, and agency), suggesting the absence of any age-related effect.

\section{Body size estimation task}

We computed the percentage of misestimation for each participant and for each body part, following Keizer et al.'s procedure $^{14}$ : percentage of misestimation $=($ estimated sizeactual size)/actual size $) \times 100$. Specifically, a negative value represented an underestimation, whereas a positive value represented an overestimation. Independent sample $t$-test indicated that there were no significant difference among the two groups in body size estimations at baseline $\left[t_{\text {shoulders }}(38)=\right.$ $0.205 ; p=0.838 ; t_{\text {abdomen }}(38)=-1.615 ; p=0.115 ; t_{\text {hips }}(38)=$

Table 1. Results Obtained from the Embodiment Questionnaire

\begin{tabular}{|c|c|c|c|c|c|c|c|}
\hline & & Age group 1 & Age group 2 & & $\mathrm{~F}$ & $\mathrm{p}$ & Partial $\eta^{2}$ \\
\hline Ownership & $\begin{array}{l}\text { Synchronous condition } \\
\text { Asynchronous condition }\end{array}$ & $\begin{array}{l}3.57(1.12) \\
3.48(1.09)\end{array}$ & $\begin{array}{l}3.78(1.41) \\
3.28(1.52)\end{array}$ & $\begin{array}{l}\text { Group } \\
\text { Condition } \\
\text { Condition } \times \text { group }\end{array}$ & $\begin{array}{l}0.001 \\
4.363 \\
1.963\end{array}$ & $\begin{array}{l}0.993 \\
0.043 \\
0.169\end{array}$ & $\begin{array}{l}0.001 \\
0.103 \\
0.049\end{array}$ \\
\hline Self-location & $\begin{array}{l}\text { Synchronous condition } \\
\text { Asynchronous condition }\end{array}$ & $\begin{array}{l}4.32(1.00) \\
3.72(1.32)\end{array}$ & $\begin{array}{l}4.46(1.47) \\
3.82(1.65)\end{array}$ & $\begin{array}{l}\text { Group } \\
\text { Condition } \\
\text { Condition } \times \text { group }\end{array}$ & $\begin{array}{r}0.090 \\
11.499 \\
0.011\end{array}$ & $\begin{array}{l}0.766 \\
0.002 \\
0.919\end{array}$ & $\begin{array}{l}0.002 \\
0.232 \\
0.001\end{array}$ \\
\hline Agency & $\begin{array}{l}\text { Synchronous condition } \\
\text { Asynchronous condition }\end{array}$ & $\begin{array}{l}4.00(1.74) \\
3.72(1.63)\end{array}$ & $\begin{array}{l}3.40(2.02) \\
3.37(1.94)\end{array}$ & $\begin{array}{l}\text { Group } \\
\text { Condition } \\
\text { Condition } \times \text { group }\end{array}$ & $\begin{array}{l}0.748 \\
0.616 \\
0.428\end{array}$ & $\begin{array}{l}0.393 \\
0.437 \\
0.517\end{array}$ & $\begin{array}{l}0.019 \\
0.016 \\
0.011\end{array}$ \\
\hline
\end{tabular}

Data are shown as mean $(S D)$. For all analyses, $\mathrm{df}=1,38$.

$S D$, standard deviation. 
Table 2. Effect of Body Swap Illusion in Inducing Changes in Body Size Estimation

\begin{tabular}{|c|c|c|c|c|c|c|}
\hline & Age group 1 & Age group 2 & & $\mathrm{~F}$ & $\mathrm{p}$ & Partial $\eta^{2}$ \\
\hline \multicolumn{7}{|l|}{ Shoulders } \\
\hline Preillusion size estimation & $-7.663(15.523)$ & $-8.715(16.875)$ & Group & 1.363 & 0.250 & 0.035 \\
\hline $\begin{array}{l}\text { Synchronous postillusion } \\
\text { size estimation }\end{array}$ & $-15.132(15.225)$ & $-4.332(17.124)$ & Condition $^{\mathrm{a}}$ & 2.036 & 1.38 & 0.051 \\
\hline $\begin{array}{l}\text { Asynchronous postillusion } \\
\text { size estimation }\end{array}$ & $-15.014(14.700)$ & $-9.802(13.644)$ & Condition $\times$ group $^{b}$ & 3.927 & 0.024 & 0.094 \\
\hline \multicolumn{7}{|l|}{ Abdomen } \\
\hline Preillusion size estimation & $-9.931(23.838)$ & $2.338(24.208)$ & Group & 4.996 & 0.031 & 0.116 \\
\hline $\begin{array}{l}\text { Synchronous postillusion } \\
\text { size estimation }\end{array}$ & $-14.909(23.484)$ & $2.365(25.395)$ & Condition $^{\mathrm{a}}$ & 1.081 & 0.344 & 0.028 \\
\hline $\begin{array}{l}\text { Asynchronous postillusion } \\
\text { size estimation }\end{array}$ & $-16.768(21.828)$ & $2.237(28.161)$ & Condition $\times$ group $^{b}$ & 1.105 & 0.337 & 0.028 \\
\hline \multicolumn{7}{|l|}{ Hips } \\
\hline Preillusion size estimation & $0.314(19.168)$ & $11.836(20.305)$ & Group & 8.902 & 0.005 & 0.190 \\
\hline $\begin{array}{l}\text { Synchronous postillusion } \\
\text { size estimation }\end{array}$ & $-3.877(19.893)$ & $18.152(26.355)$ & Condition $^{\mathrm{a}}$ & 1.660 & 0.197 & 0.042 \\
\hline $\begin{array}{l}\text { Asynchronous postillusion } \\
\text { size estimation }\end{array}$ & $-6.666(18.047)$ & 13.238 (18.048) & Condition $\times$ group $^{\mathrm{b}}$ & 3.237 & 0.045 & 0.078 \\
\hline
\end{tabular}

Data are shown as mean $(S D)$.

${ }^{\mathrm{a}} \mathrm{df}=1,38$.

$\mathrm{b} d f=2,76$.

$-1.845 ; p=0.073]$. According to a mixed ANOVA relative to the shoulders width estimation, no main effect of condition or group emerged. Importantly, the interaction between condition and group was significant (Table 2). Bonferroni-corrected pairwise comparisons revealed that 19-to-25-year-old participants significantly underestimated shoulders width in the synchronous postillusion condition compared with the preillusion-baseline phase $(p=0.05)$ (Table 3$)$. No other comparisons resulted significant.

Table 3. Interaction Between Condition AND GROUP FOR SHOULDERS Estimates

\begin{tabular}{|c|c|c|c|}
\hline & $\begin{array}{c}\text { Mean difference } \\
(I-J)\end{array}$ & $\begin{array}{l}\text { Standard } \\
\text { error }\end{array}$ & $\mathrm{p}$ \\
\hline \multicolumn{4}{|l|}{ Age group 1} \\
\hline \multicolumn{4}{|c|}{ Preillusion size estimation } \\
\hline Synchronous & 7.469 & 2.978 & 0.050 \\
\hline Asynchronous & 7.351 & 3.082 & 0.066 \\
\hline \multicolumn{4}{|c|}{ Synchronous postillusion size estimation } \\
\hline Preillusion & -7.469 & 2.978 & 0.050 \\
\hline Asynchronous & -0.118 & 2.915 & 1 \\
\hline \multicolumn{4}{|c|}{ Asynchronous postillusion size estimation } \\
\hline Preillusion & -7.351 & 3.082 & 0.066 \\
\hline Synchronous & 0.118 & 2.915 & 1 \\
\hline \multicolumn{4}{|l|}{ Age group 2} \\
\hline \multicolumn{4}{|c|}{ Preillusion size estimation } \\
\hline Synchronous & -4.383 & 2.978 & 0.448 \\
\hline Asynchronous & 1.087 & 3.082 & 1 \\
\hline \multicolumn{4}{|c|}{ Synchronous postillusion size estimation } \\
\hline Preillusion & 4.384 & 2.978 & 0.448 \\
\hline Asynchronous & 5.471 & 2.915 & 0.205 \\
\hline \multicolumn{4}{|c|}{ Asynchronous postillusion size estimation } \\
\hline Preillusion & -1.087 & 3.082 & 1 \\
\hline Synchronous & -5.471 & 2.915 & 0.205 \\
\hline
\end{tabular}

Bonferroni pairwise comparisons.
Referring to the abdomen width estimation, a main effect of group emerged, but not of condition neither a significant interaction (Table 2). This means that, regardless of condition, 19-to-25-year-old participants (estimates mean = -13.869 ; standard error $=5.134$ ) reported a larger percentage of misestimation for abdomen, underestimating its size, in comparison with 26-to-55-year-old participants (estimates mean $=2.359$; standard error $=5.134$ ). Finally, about hips, a main effect of group and a significant interaction between condition and group (Table 2) emerged. Participants of

Table 4. Interaction Between Condition AND GROUP FOR HIPS ESTIMATES

\begin{tabular}{|c|c|c|c|}
\hline & $\begin{array}{c}\text { Mean } \\
\text { difference }(I-J)\end{array}$ & $\begin{array}{l}\text { Standard } \\
\quad \text { error }\end{array}$ & $\mathrm{p}$ \\
\hline \multicolumn{4}{|l|}{ Age group 1} \\
\hline \multicolumn{4}{|c|}{ Preillusion size estimation } \\
\hline Synchronous & 4.191 & 3.290 & 0.632 \\
\hline Asynchronous & 6.980 & 2.674 & 0.039 \\
\hline \multicolumn{4}{|c|}{ Synchronous postillusion size estimation } \\
\hline Preillusion & -4.191 & 3.290 & 0.632 \\
\hline Asynchronous & 2.789 & 3.260 & 1 \\
\hline \multicolumn{4}{|c|}{ Asynchronous postillusion size estimation } \\
\hline Preillusion & -6.980 & 2.674 & 0.039 \\
\hline Synchronous & -2.789 & 3.260 & 1 \\
\hline \multicolumn{4}{|l|}{ Age group 2} \\
\hline \multicolumn{4}{|c|}{ Preillusion size estimation } \\
\hline Synchronous & -6.316 & 3.290 & 0.187 \\
\hline Asynchronous & -1.402 & 2.674 & 1 \\
\hline \multicolumn{4}{|c|}{ Synchronous postillusion size estimation } \\
\hline Preillusion & 6.316 & 3.290 & 0.187 \\
\hline Asynchronous & 4.915 & 3.260 & 0.419 \\
\hline \multicolumn{4}{|c|}{ Asynchronous postillusion size estimation } \\
\hline Preillusion & 1.402 & 2.673 & 1 \\
\hline Synchronous & -4.915 & 3.260 & 0.420 \\
\hline
\end{tabular}

Bonferroni pairwise comparisons. 
26-to-55-year old showed again a larger percentage of misestimation (estimates mean $=14.409$; standard error $=$ 4.223) in comparison with 19-to-25-year-old participants (estimates mean $=-3.410$; standard error $=4.223$ ). Interestingly, Bonferroni-corrected pairwise comparisons revealed that 19-to-25-year-old participants' misestimation of hips width significantly increases from preillusion to asynchronous postillusion $(p=0.039)$ (Table 4$)$. Although 26-to-55year-old participants showed a tendency to overestimate, 19to-25-year-old participants exhibited the opposite tendency (i.e., the underestimation), which increased after the illusion. No other comparisons were significant.

\section{Discussion}

The aim of this study was to explore possible age-related differences in body representation changes after a VR fullbody illusion. Results from the Embodiment Questionnaire ${ }^{1,22}$ indicated that the feeling of owning a virtual body and being in the same place of the virtual body were higher in the synchronous condition for all participants. This means that, regardless of age, participants reported to experience the illusion in terms of body ownership and self-location, but not in terms of agency, as expected since the avatar was static. Our findings revealed that, independently of the type of stimulation, 19-to25-year-old participants globally reported an increase of underestimation after embodying a virtual body with a skinny abdomen (especially for shoulders and hips), whereas 26-to55-year-old participants appeared more resistant to changes of their body perception after the bodily illusion.

To date, very few studies, with contrasting results, have explored possible age-related differences in responses to the bodily illusions. Some experimental studies using the "rubber hand illusion" (RHI) (i.e., the prototypical paradigm of the class of bodily illusions, full-body illusion included ${ }^{23}$ ) have reported that children show a larger proprioceptive drift toward the fake hand in comparisons with young adults, ${ }^{24,25}$ but this difference is not mirrored in their explicit feeling of ownership. ${ }^{24}$ Kállai et al. ${ }^{26}$ recently found that old participants (mean age: 65.9 years old) reported less vivid ownership toward the rubber hand compared with younger participants (mean age: 27.7 years old).

A very recent study carried out by Palomo et al. ${ }^{27}$ did not find any differences among three different target groups (2035 years old, 36-60 years old, and 61-80 years old) in response to the RHI, suggesting that this illusion elicits the same "embodiment process" across different ages. Two studies specifically focused on middle-aged participants, that is, a target group similar to our study. Tajadura-Jiménez et al. $^{28}$ used the so-called enfacement illusion, that is, the delivery of a synchronous multisensory stimulation between one's own face and another person's face to induce changes in self-identification. They found that middle-age participants were more resistant to the illusion than younger participants; it might be that the huge changes in visual body appearance in the middle age require a larger plasticity of body representation.

Using a variant of the RHI, Graham et al. ${ }^{29}$ found a decrease in the perception of the illusion with age. These authors introduced a Bayesian mechanism to explain how illusion works: it is possible to experience two spatially congruent perceptions as linked thanks to prior probabilities associated with one's own history of perceptual experiences. This results in the illusory perception of the "fake" hand as one's own hand. With increasing age, individuals have a great amount of experience of spatially congruent perceptions, thus it might decrease the prior probabilities of two spatially incongruent perceptions as originating from one's own hand, reducing the possibility of experiencing the illusion. Both these two interpretations can be embraced to explain the lower level of plasticity of 26-to-55-year-old participants' body representation. Piryankova et al. ${ }^{22}$ reported that their participants changed the "experienced body" (in their terminology, "the body that the participant feels he/she has at that moment") before any type of stimulation, but only when they viewed an underweighted (and not an overweighted) virtual body. They discussed these findings arguing that, beyond the role of visual input from seeing the virtual body, participants probably used the "memory" of their own body" ${ }^{30-33}$ for estimating the physical body dimensions.

Accordingly, in our experiment, the illusion might affect body perception in 19-to-25-year-old participants, but not that of the 26-to-55-year-old participants, since their body representation (stored in memory ${ }^{33}$ ) was more stable. During aging, there is a shift in cortical responses from sensory regions to executive regions (the so-called compensation hypothesis ${ }^{34}$ ). According to a Bayesian approach, ${ }^{35-37}$ the relative "lower plasticity" of the body representation in 26to-55-year-old participants could be interpreted as a more rigid use of predictive strategies acquired during the lifespan (as observed also by Graham et al. ${ }^{29}$ ) instead of adapting these strategies to sensory inputs. ${ }^{38}$ However, future research to support this suggestion is required.

It is crucial to note that the difference between the two groups emerged independently from the conditions, thus suggesting that solely viewing a virtual body while it was stimulating can modify the body perception for 19-to-25year-old participants. It was likely that participants experienced this illusion also in the asynchronous condition, even at less extent. Indeed, it has already been demonstrated that a first-person perspective (i.e., an avatar being spatially coincident with the position of the participant) of a realistic virtual body substituting participants' own body is sufficient to generate an illusory feeling of ownership and changes in body representations, ${ }^{1}$ even though after an asynchronous stimulation. $1,10,11$

In conclusion, this study represents the first attempt to provide evidence about the effect of age on multisensory bodily experience offering valuable insights to guide and stimulate future research in this area. In particular, it may stimulate research investigating possible clinical application of bodily illusions ${ }^{13,16}$ for shaping body perception also in eating disorders among women in midlife. ${ }^{39}$

However, despite these promising results, some limitations arose. First, it should be noted that we primarily opted for only a self-report measure of embodiment, although it would have of interest to have also physiological (i.e., skin conductance response or body temperature) measurements. Second, future studies should investigate the effect of different ages, ranging from 18-year-olds to elderly individuals, as in the study of Palomo et al. ${ }^{27}$ Third, it should be acknowledged that our sample counted only female participants, thus current findings cannot be spread also to male 
population. Finally, it should be noted that the second group included a larger life span period-women older than 35 years-with a reduced probability of pregnancy. ${ }^{40}$

Future studies should use different avatars of different ages and investigate the role of psychological factors (i.e., body esteem and self-esteem) and include also the use of simulation/stimulation technologies able to modulate the inner experience of the body ${ }^{41}$ to fully capture the complicated patterns of age and body representations across lifespan.

\section{Notes}

a. www.unity3d.com

b. www.makehuman.org/

\section{Acknowledgments}

The authors thank Ordina ICT B. V for technical development of the VR-Body Swap Illusion. This work was partly supported by the research project "Ageing and Healthy Living: A Human Centered Approach in Research and innovation as Source of Quality Life," funded by Fondazione Cariplo within 2014 and by the Italian MIUR PRIN research project "Unlocking the memory of the body: Virtual Reality in Anorexia Nervosa", (201597WTTM).

\section{Author Disclosure Statement}

No competing financial interests exist.

\section{References}

1. Serino S, Pedroli E, Keizer A, et al. Virtual reality body swapping: a tool for modifying the allocentric memory of the body. Cyberpsychology, Behavior, and Social Networking 2016; 19:127-133.

2. Normand JM, Giannopoulos E, Spanlang B, et al. Multisensory stimulation can induce an illusion of larger belly size in immersive virtual reality. PLoS One 2011; 6: e16128.

3. Preston C, Ehrsson HH. Illusory changes in body size modulate body satisfaction in a way that is related to nonclinical eating disorder psychopathology. PLoS One 2014; 9:e85773.

4. Costantini M. Body perception, awareness, and illusions. Wiley Interdisciplinary Reviews: Cognitive Science 2014; 5:551-560.

5. Dieguez S, Lopez C. The bodily self: insights from clinical and experimental research. Annals of Physical and Rehabilitation Medicine 2017; 60:198-207.

6. Brugger P, Lenggenhager B. The bodily self and its disorders: neurological, psychological and social aspects. Current Opinion in Neurology 2014; 27:644-652.

7. Blanke O, Slater M, Serino A. Behavioral, neural, and computational principles of bodily self-consciousness. Neuron 2015; 88:145-166.

8. Kilteni K, Maselli A, Kording KP, et al. Over my fake body: body ownership illusions for studying the multisensory basis of own-body perception. Frontiers in Human Neuroscience 2015; 9:141.

9. Petkova VI, Ehrsson HH. If I were you: perceptual illusion of body swapping. PLoS One 2008; 3:e3832.

10. Maselli A, Slater M. The building blocks of the full body ownership illusion. Frontiers in Human Neuroscience 2013; $7: 83$.
11. Slater M, Spanlang B, Sanchez-Vives MV, et al. First person experience of body transfer in virtual reality. PLoS One 2010; 5:e10564.

12. Dakanalis A, Gaudio S, Serino S, et al. Body-image distortion in anorexia nervosa. Nature Reviews Disease Primers 2016; 2:16026.

13. Serino S, Dakanalis A. Bodily illusions and weight-related disorders: clinical insights from experimental research. Annals of Physical and Rehabilitation Medicine 2017; 60: 217-219.

14. Keizer A, van Elburg A, Helms R, et al. A virtual reality full body illusion improves body image disturbance in anorexia nervosa. PLoS One 2016; 11:e0163921.

15. Serino S, Scarpina F, Keizer A, et al. A novel technique for improving bodily experience in a non-operable super-super obesity case. Frontiers in Psychology 2016; 7:837.

16. Riva G, Baños RM, Botella C, et al. Transforming experience: the potential of augmented reality and virtual reality for enhancing personal and Clinical change. Frontiers in Psychiatry 2016; 7:164.

17. Ferrer-Garcia M, Gutiérrez-Maldonado J, Riva G. Virtual reality based treatments in eating disorders and obesity: a review. Journal of Contemporary Psychotherapy 2013; 43: 207-221.

18. Wiederhold BK, Riva G, Gutiérrez-Maldonado J. Virtual reality in the assessment and treatment of weight-related disorders. Cyberpsychology, Behavior, and Social Networking 2016; 19:67-73.

19. Gutiérrez-Maldonado J, Wiederhold BK, Riva G. Future directions: how virtual reality can further improve the assessment and treatment of eating disorders and obesity. Cyberpsychology, Behavior, and Social Networking 2016; 19:148-153.

20. Riva G. Letter to the Editor: virtual reality in the treatment of eating and weight disorders. Psychological Medicine 2017; 47:2567.

21. American Psychiatric Association. (2000). Diagnostic and statistical manual of mental disorders-IV-TR. Washington, DC: American Psychiatric Association.

22. Piryankova IV, Wong HY, Linkenauger SA, et al. Owning an overweight or underweight body: distinguishing the physical, experienced and virtual body. PLoS One 2014; 9: e103428.

23. Botvinick M, Cohen J. Rubber hands' feel'touch that eyes see. Nature 1998; 391:756.

24. Cowie D, Makin TR, Bremner AJ. Children's responses to the rubber-hand illusion reveal dissociable pathways in body representation. Psychological Science 2013; 24:762-769.

25. Bremner AJ, Hill EL, Pratt M, et al. Bodily illusions in young children: developmental change in visual and proprioceptive contributions to perceived hand position. PLoS One 2013; 8:e51887.

26. Kállai J, Kincses P, Lábadi B, et al. Multisensory integration and age-dependent sensitivity to body representation modification induced by the rubber hand illusion. Cognitive Processing 2017; 18:349-357.

27. Palomo P, Borrego A, Cebolla A, et al. Subjective, behavioral, and physiological responses to the rubber hand illusion do not vary with age in the adult phase. Consciousness and Cognition 2018; 58:90-96.

28. Tajadura-Jiménez A, Longo MR, Coleman R, et al. The person in the mirror: using the enfacement illusion to investigate the experiential structure of self-identification. Consciousness and Cognition 2012; 21:1725-1738. 
29. Graham KT, Martin-Iverson MT, Holmes NP, et al. The projected hand illusion: component structure in a community sample and association with demographics, cognition, and psychotic-like experiences. Attention, Perception, and Psychophysics 2015; 77:207-219.

30. Longo MR, Azañón E, Haggard P. More than skin deep: body representation beyond primary somatosensory cortex. Neuropsychologia 2010; 48:655-668.

31. Riva G. Out of my real body: cognitive neuroscience meets eating disorders. Frontiers in Human Neuroscience 2014; 8: 236.

32. Riva G. Neurobiology of anorexia nervosa: serotonin dysfunctions link self-starvation with body image disturbances through an impaired body memory. Frontiers in Human Neuroscience 2016; 10:600.

33. Riva G. The neuroscience of body memory: from the self through the space to the others. Cortex 2017. [Epub ahead of print] DOI: 10.1016/j.cortex.2017.07.013

34. Cabeza R. Hemispheric asymmetry reduction in older adults: the HAROLD model. Psychology and Aging 2002; 17:85.

35. Friston K. The free-energy principle: a unified brain theory? Nature Reviews Neuroscience 2010; 11:127-138.

36. Tsakiris M. My body in the brain: a neurocognitive model of body-ownership. Neuropsychologia 2010; 48:703-712.

37. Apps MAJ, Tsakiris M. The free-energy self: a predictive coding account of self-recognition. Neuroscience and Biobehavioral Reviews 2014; 41:85-97.
38. Moran RJ, Symmonds M, Dolan RJ, et al. The brain ages optimally to model its environment: evidence from sensory learning over the adult lifespan. PLoS Computational Biology 2014; 10:e1003422.

39. Lewis-Smith H, Diedrichs PC, Rumsey N, et al. A systematic review of interventions on body image and disordered eating outcomes among women in midlife. International Journal of Eating Disorders 2016; 49:5-18.

40. Dunson DB, Baird DD, Colombo B. Increased infertility with age in men and women. Obstetrics and Gynecology 2004; 103:51-56.

41. Riva G, Serino S, Di Lernia D, et al. Embodied medicine: mens sana in corpore virtuale sano. Frontiers in Human Neuroscience 2017; 11:120.

Address correspondence to: Dr. Silvia Serino Department of Psychology Università Cattolica del Sacro Cuore Largo Gemelli, 1 Milan 20100 Italy

E-mail: s.serino@auxologico.it 